\title{
Non-randomised patients in a cholecystectomy trial: characteristics, procedures, and outcomes Axel Ros ${ }^{1}$, Per Carlsson², Mikael Rahmqvist ${ }^{2}$, Karin Bäckman² and Erik Nilsson*3
}

\author{
Address: ${ }^{1}$ Department of Surgery, County Hospital of Ryhov, Jönköping, Sweden, ${ }^{2}$ Center for Medical Technology Assessment, Linköping \\ University, Linköping, Sweden and ${ }^{3}$ Department of Surgery, University Hospital of Umeå, Sweden \\ Email: Axel Ros - axel.ros@lj.se; Per Carlsson - per.carlsson@ihs.liu.se; Mikael Rahmqvist - mikra@ihs.liu.se; \\ Karin Bäckman - Karin.Backman@lio.se; Erik Nilsson* - erik.nilsson@surgery.umu.se \\ * Corresponding author
}

Published: 26 December 2006

BMC Surgery 2006, 6:17 doi:10.1186/147/-2482-6-17

This article is available from: http://www.biomedcentral.com/I47/-2482/6/17

(C) 2006 Ros et al; licensee BioMed Central Ltd.

This is an Open Access article distributed under the terms of the Creative Commons Attribution License (http://creativecommons.org/licenses/by/2.0), which permits unrestricted use, distribution, and reproduction in any medium, provided the original work is properly cited.

\begin{abstract}
Background: Laparoscopic cholecystectomy is now considered the first option for gallbladder surgery. However, $20 \%$ to $30 \%$ of cholecystectomies are completed as open operations often on elderly and fragile patients. The external validity of randomised trials comparing mini-laparotomy cholecystectomy and laparoscopic cholecystectomy has not been studied. The aim of this study is to analyse characteristics, procedures, and outcomes for all patients who underwent cholecystectomy without being included in such a trial.
\end{abstract}

Methods: Characteristics (age, sex, co-morbidity, and ASA-score), operation time, hospital stay, and mortality were compared for patients who underwent cholecystectomy outside and within a randomised controlled trial comparing mini-laparotomy and laparoscopic cholecystectomy.

Results: During the inclusion period 1719 patients underwent cholecystectomy. 726 patients were randomised and 724 of them completed the trial; 993 patients underwent cholecystectomy outside the trial. The non-randomised patients were older - and had more complications from gallstone disease, higher co-morbidity, and higher ASA - score when compared with trial patients. They were also more likely to undergo acute surgery and they had a longer postoperative hospital stay, with a median 3 versus 2 days ( $p<0.00$ I for all comparisons). Standardised mortality ratio within 90 days of operation was 3.42 (mean) $(95 \% \mathrm{Cl} 2.17$ to 5.13$)$ for non-randomised patients and I.6I (mean) $(95 \% \mathrm{Cl} 0.02$ to 3.46$)$ for trial patients. For non-randomised patients, operation time did not differ significantly between mini-laparotomy and open cholecystectomy in multivariate analysis. However, the operation for laparoscopic cholecystectomy lasted 20 minutes longer than open cholecystectomy. Hospital stay was significantly shorter for both mini-laparotomy and laparoscopic cholecystectomy compared to open cholecystectomy.

Conclusion: Non-randomised patients were older and more sick than trial patients. The assignment of healthier patients to trials comparing mini-laparotomy cholecystectomy and laparoscopic cholecystectomy limits the external validity of conclusions reached in such trials. 


\section{Background}

External validity of conclusions reached in a randomised trial depends upon the number and characteristics of eligible patients not included in the trial (excluded by patient or researcher) and number and characteristics of patients who do not meet the eligibility criteria [1]. According to the CONSORT [2] recommendations, the external validity of a study should be reported. However, in surgical trials, there is often a lack of data concerning the pre-randomisation collection of patients.

The laparoscopic technique has been adopted as the method of choice for cholecystectomy, and epidemiological studies indicate that $70 \%$ to $80 \%$ of all cholecystectomies are now completed laparoscopically [3-5]. However, in single-blind, randomised controlled trials the difference in convalescence between small-incision open surgery (mini-laparotomy cholecystectomy) and laparoscopic cholecystectomy is small and of short duration $[6,7]$. No previous study has reported data on nonrandomised patients. The present report describes characteristics, operation time, postoperative hospital stay, and postoperative mortality of all non-randomised cholecystectomy patients not included in a randomised controlled trial [7].

\section{Methods \\ Patients}

Data for all patients over age 18 advised to undergo elective or acute cholecystectomy were prospectively recorded at four non-university hospitals and one university centre. Patients undergoing cholecystectomy as adjunct to other major abdominal operations such as pancreatic resection, hepatic resection and aneurysmectomy were excluded. The protocol included information on previous and present cholecystitis, pancreatitis, jaundice, and suspected or proven malignancy; American Society of Anaesthesiologists' (ASA) score; co-morbidity (heart disease, lung disease, liver/renal disease, diabetes with insulin treatment, steroid therapy); diagnostic investigations; and indication for surgery (elective/acute).

\section{Trial}

Eligible patients were considered for inclusion in a randomised controlled trial comparing minilaparotomy and laparoscopic cholecystectomy. Patients were given verbal and written information about the trial and asked if they wished to participate. Exclusion criteria for the trial were: age under 18 years, jaundice, obesity (body mass index $>45$ ), pregnancy, cirrhosis of the liver, suspected or proven malignancy, and previous upper gastrointestinal surgery. Recruitment of patients, randomisation, blinding, sample size calculation, control panel and rules for stopping the trial, data collection, and processing have been described in previous reports $[7,8]$. During the inclu- sion period, 1705 cholecystectomy patients were identified. Scrutiny of medical records yielded another 14 nonrandomised patients. 726 patients were randomised, but two randomised patients were withdrawn: one patient unexpectedly had disseminated malignancy found at laparoscopy and the other patient was randomised to laparoscopic cholecystectomy but was operated with minilaparotomy for unknown reasons. This change was detected too late in data handling to allow collection of necessary information. Analysis was otherwise performed on an "intention-to-treat" basis for both randomised and non-randomised patients. Thus, a converted laparoscopic operation was classified as laparoscopic cholecystectomy and mini-laparotomy cholecystectomy with extended incision (beyond $8 \mathrm{~cm}$ ) as mini-laparotomy cholecystectomy. 277 patients were excluded from the trial according to protocol (exclusion criteria), 472 patients were excluded by surgeons in charge, and 244 patients, did not want to participate in the trial. Thus, characteristics and outcomes for 993 non-randomised patients and 724 randomised patients could be compared. Approval of the Ethics Committee was obtained.

\section{Surgery}

Laparoscopic cholecystectomy was performed according to routines at the participating units. Mini-laparotomy was defined as cholecystectomy performed through a laparotomy incision less than $8 \mathrm{~cm}$. Trainees were encouraged to participate in surgery under supervision, as is routine practice. Complications were classified according to Clavien et al [9].

\section{Statistics}

Outcome of qualitative variables, for example acute cholecystitis (yes/no) or co-morbidity (no/at least one disease or item) was compared using chi-square analysis. The distribution of quantitative variables (e.g. operating time, hospital stay) was compared using the Kolmogorov-Smirnov non-parametric two-sample test. Mortality within 90 days and from 91 to 365 days of surgery was calculated as the standardised mortality ratio (SMR) i. e. ratio between the observed and expected number of deceased patients, taking into account sex and age of individuals. Mortality after cholecystectomy in a defined population is raised above that of the standard population until 90 days after operation [4] and therefore this postoperative period was used for assessment of postoperative mortality.

Among non-randomised patients, operation time and hospital stay for open cholecystectomy, mini-laparotomy cholecystectomy, and laparoscopic cholecystectomy were compared using multiple regression analysis with open cholecystectomy as a reference. First, the influence of acute cholecystitis, pancreatitis, jaundice, malignancy, comorbidity, ASA-score, common bile duct exploration, 
bilio-digestive shunt operation (choledocho-duodenostomy), concomitant operation, and acute operation was tested using univariate analyses. Adjustments were then made for these variables in a multiple regression analysis.

\section{Results}

\section{Non-randomised versus randomised patients}

Table 1 shows the characteristics of trial patients and nonrandomised patients. The gender distribution did not differ between the two groups. Trial patients were younger and less likely to have acute operations, complications of gallstone disease, associated diseases, and ASA scores III and IV. Malignancy was identified in 27 of 993 non-randomised patients, $2.7 \%$.

The procedures used for non-randomised patients are shown in Table 2. 52.4\% of these patients were treated with conventional open cholecystectomy, $15.5 \%$ with mini-laparotomy, and $32.1 \%$ with laparoscopic cholecystectomy. The trial protocol did not exclude concomitant operations, but these were more common among nonrandomised patients $(6.9 \%$ vs $2.8 \%)$. Operation time, reoperation rate, and re-admissions did not differ between trial patients and non-randomised patients, whereas nonrandomised patients had a longer postoperative stay ( $\mathrm{p}<$ 0.001) (Table 3). As shown in Table 4, the risk of death for non-randomised patients significantly exceeded that of the standard population within 90 days of surgery.

\section{Operation time and postoperative hospital stay in non- randomised patients}

According to multiple regression analysis, operation time for mini-laparotomy cholecystectomy and open cholecystectomy in non-randomised patients did not differ significantly, whereas laparoscopic cholecystectomy took significantly longer (20 minutes) than open cholecystectomy (Table 5). Hospital stay was significantly shorter for both mini-laparotomy cholecystectomy and laparoscopic cholecystectomy compared to open cholecystectomy.

\section{Discussion}

The principal findings of present study are that non-randomised patients were older and more sick than trial patients and more likely to have an acute operation. Of all non-randomised patients $67.9 \%$ were operated with open methods, $52.4 \%$ with conventional open cholecystectomy, and $15.5 \%$ with mini-laparotomy cholecystectomy. In contrast to trial patients, non-randomised patients had a threefold excess death rate compared to the background population during the postoperative period. Multivariate analyses demonstrated that operation time for non-randomised patients did not differ between open cholecystectomy and mini-laparotomy, whereas laparoscopic cholecystectomy took 20 minutes longer. Postoperative hospital stay was shorter for both mini-laparotomy cholecystectomy and laparoscopic cholecystectomy compared to open cholecystectomy.

The strength of the study is the completeness of the data. All cholecystectomies performed during the inclusion period are taken into account. The weaknesses of the study primarily relate to timing. Our study was conducted at a time when laparoscopic cholecystectomy had been in use for five years in Sweden and surgeons' experience with mini-laparotomy was limited. Cholecystectomy was not performed as day-case surgery at the time of the trial, but day-cases have since been introduced both for laparoscopic cholecystectomy and mini-laparotomy at the hospitals participating in the present trial.

Cholecystectomy in elderly and vulnerable patients, who often have complications of gallstone disease, is associated with considerable mortality $[5,10]$. Therefore, it is an expected observation that our non-randomised patients had a postoperative mortality three times that of the standard population. Whereas $32 \%$ of all cholecystectomies performed from 1995 to 1999 in Sweden for acute or chronic gallbladder disease were completed as open operations, the majority of patients over age 70 had an open operation [5]. Under these circumstances, the high percentage of open cholecystectomy among our non-randomised patients is not surprising. Nevertheless, the number of conventional open cholecystectomy compared to mini-laparotomy cholecystectomy is a matter of concern, as randomised controlled trials [11-13] have concluded that the surgical trauma is greater after conventional open cholecystectomy than after minilaparotomy cholecystectomy.

Table I: Characteristics of trial patients and non-randomised patients

\begin{tabular}{|c|c|c|c|c|c|c|c|c|c|c|c|c|c|c|c|c|c|c|c|}
\hline & \multirow[b]{2}{*}{$\mathbf{n}$} & \multicolumn{2}{|c|}{ Women } & \multicolumn{2}{|c|}{ Age, years } & \multicolumn{2}{|c|}{$\begin{array}{c}\text { Acute } \\
\text { operation }\end{array}$} & \multicolumn{2}{|c|}{$\begin{array}{c}\text { Acute } \\
\text { cholecystitis }\end{array}$} & \multicolumn{2}{|c|}{ Pancreatitis } & \multicolumn{2}{|c|}{ Jaundice } & \multicolumn{2}{|c|}{ Malignancy } & \multicolumn{2}{|c|}{$\begin{array}{c}\text { Associated } \\
\text { diseases }\end{array}$} & \multicolumn{2}{|c|}{ ASA III+IV } \\
\hline & & $\mathbf{n}$ & $\%$ & Mean & SD & $\mathbf{n}$ & $\%$ & $\mathbf{n}$ & $\%$ & $\mathbf{n}$ & $\%$ & $\mathbf{n}$ & $\%$ & $\mathbf{n}$ & $\%$ & $\mathbf{n}$ & $\%$ & $\mathbf{n}$ & $\%$ \\
\hline Trial patients & 724 & 501 & 69.2 & 50.6 & 15.6 & 106 & 14.6 & 72 & 8.6 & 0 & & 0 & & 0 & & 154 & 21.3 & 28 & 3.9 \\
\hline \multirow{2}{*}{$\begin{array}{l}\text { Non-randomised } \\
\text { patients }\end{array}$} & 993 & 657 & 66.2 & 56.5 & 19.1 & 516 & 52.0 & 405 & 40.8 & 53 & 5.4 & 140 & 14.1 & 27 & 2.7 & 336 & 33.8 & 99 & 14.1 \\
\hline & & \multicolumn{2}{|c|}{$p=0.18$} & \multicolumn{2}{|c|}{$p<0.001$} & \multicolumn{2}{|c|}{$p<0.001$} & \multicolumn{2}{|c|}{$\mathrm{p}<0.001$} & \multicolumn{2}{|c|}{$p<0.001$} & \multicolumn{2}{|c|}{$p<0.001$} & \multicolumn{2}{|c|}{$p<0.001$} & \multicolumn{2}{|c|}{$p<0.001$} & \multicolumn{2}{|c|}{$p<0.001$} \\
\hline
\end{tabular}


Table 2: Operations on trial patients and non-randomised patients

\begin{tabular}{|c|c|c|c|c|c|c|c|c|c|c|c|c|c|}
\hline & \multirow[b]{2}{*}{$\mathbf{n}$} & \multicolumn{2}{|c|}{ OC } & \multicolumn{2}{|c|}{ LC } & \multicolumn{2}{|c|}{ MC } & \multicolumn{2}{|c|}{ CBDE } & \multicolumn{2}{|c|}{ Shunt } & \multicolumn{2}{|c|}{ Concomitant operation } \\
\hline & & $\mathbf{N}$ & $\%$ & $\mathbf{n}$ & $\%$ & $\mathbf{n}$ & $\%$ & $\mathbf{n}$ & $\%$ & $\mathbf{n}$ & $\%$ & $\mathbf{N}$ & $\%$ \\
\hline Trial patients & 724 & 0 & & 362 & 50.0 & 362 & 50.0 & 39 & 5.4 & 0 & & 20 & 2.8 \\
\hline \multirow{2}{*}{ Non-randomised patients } & 993 & 520 & 52.4 & 319 & 32.1 & 154 & 15.5 & 201 & 20.2 & 15 & 1.5 & 69 & 6.9 \\
\hline & & & & & & & & \multicolumn{2}{|c|}{$p<0.001$} & $\mathrm{p}<0$ & .001 & \multicolumn{2}{|c|}{$P<0.001$} \\
\hline
\end{tabular}

$\mathrm{OC}=$ conventional open cholecystectomy, $\mathrm{LC}=$ Laparoscopic cholecystectomy, $\mathrm{MC}=$ mini-laparotomy cholecystectomy, CBDE $=$ common bile duct exploration, Shunt $=$ choledochoduodenostomy, Concomitant operation $=$ other operation performed in combination with cholecystectomy.

Table 3: Operation time, postoperative stay, and re-operation of trial patients and non-randomised patients

\begin{tabular}{|c|c|c|c|c|c|c|c|c|c|c|c|}
\hline & \multirow[t]{2}{*}{$\mathbf{N}$} & \multicolumn{2}{|c|}{ Operation time, $\min$} & \multicolumn{4}{|c|}{ Postoperative stay, days } & \multicolumn{2}{|c|}{ Re-operation } & \multicolumn{2}{|c|}{ Re-admitted within 30 days } \\
\hline & & Mean & SD & Mean & Median & Percentiles & & $\mathbf{N}$ & $\%$ & $\mathbf{N}$ & $\%$ \\
\hline & & & & & & $25 \%$ & $75 \%$ & & & & \\
\hline Trial patients & 724 & 101 & 45 & 2.6 & 2 & 1 & 3 & 17 & 2.3 & 47 & 6.5 \\
\hline \multirow{2}{*}{$\begin{array}{l}\text { Non-randomised } \\
\text { patients }\end{array}$} & 993 & 100 & 47 & 4.9 & 3 & 2 & 6 & 23 & 2.3 & 60 & 6.1 \\
\hline & & \multicolumn{2}{|c|}{$P=0.67$} & \multicolumn{4}{|c|}{$P<0.001$} & \multicolumn{2}{|c|}{$p=0.92$} & \multicolumn{2}{|r|}{$p=0.71$} \\
\hline
\end{tabular}

Table 4: Standardised mortality ratio (SMR), mean and $(95 \% \mathrm{Cl})$

\begin{tabular}{|c|c|c|c|c|c|}
\hline & & \multicolumn{2}{|r|}{0 - 90 days } & \multicolumn{2}{|r|}{91 - 365 days } \\
\hline & $\mathbf{N}$ & Deaths & SMR & Deaths & SMR \\
\hline Trial patients & 724 & I & $1.61(0.02-3.46)$ & 3 & $0.62(0.13-1.78)$ \\
\hline Non-randomised patients & 993 & 23 & $3.42(2.17-5.13)$ & 26 & $1.27(0.90-1.86)$ \\
\hline
\end{tabular}

Table 5: Operation time and postoperative hospital stay for non-randomised patients according to multiple regression analysis

\begin{tabular}{lll}
\hline \multicolumn{1}{c}{ Operation time* $\min$} & Postoperative hospital stay** Days \\
\hline$M C-O C$ & $-2.7 p=0.50$ & $-1.3 p=0.004$ \\
LC - OC & $20.2 p<0.001$ & $-1.2 p=0.003$
\end{tabular}

$M C=$ minilaparotomy cholecystectomy, $L C=$ laparoscopic cholecystectomy, and $O C=$ conventional open cholecystectomy. Figures indicate adjusted differences between MC or LC and OC.

*Operation time adjusted for common bile duct exploration and concomitant operation.

**Postoperative hospital stay adjusted for pancreatitis, malignant disease, common bile duct exploration, biliodigestive shunt operation, comorbidity, ASA-score, concomitant operation, and acute operation.

Our multiple regression analyses indicated that minilaparotomy was a cost-effective alternative to laparoscopic cholecystectomy among non-randomised patients. This finding agrees with findings in single-blind randomised controlled trials $[8,14]$. and observational studies $[15,16]$ demonstrating low health care costs for mini-laparotomy cholecystectomy. The effectiveness of an operation can only be assessed on an unselected set of patients and only when surgery has been performed as part of the normal hospital routine. Outcomes after mini-laparotomy cholecystectomy that are comparable to outcomes after laparoscopic cholecystectomy have been reported from units/ surgeons where mini-laparotomy is the standard procedure [15-17] or regularly practised $[18,19]$.

\section{Conclusion}

We found that non-randomised patients were more vulnerable than trial patients. Mini-laparotomy should 
replace conventional open cholecystectomy whenever possible. The versatility of mini-laparotomy has important implications for cost-effectiveness in gallbladder surgery. Low external validity of conventional randomised controlled trials as demonstrated in this report may to a great extent be overcome by expertise based randomised trials [20].

\section{Competing interests}

The author(s) declare that they have no competing interests.

\section{Authors' contributions}

$\mathrm{AR}, \mathrm{EN}$, and $\mathrm{PC}$ designed the study; $\mathrm{AR}$ was responsible for collection of clinical data; and PC, MR, and $\mathrm{KB}$ for data analyses. EN drafted the manuscript. All authors read and approved the final manuscript which has been approved by all authors.

\section{Acknowledgements}

Surgeons responsible for this study at other participating centres are Hans Krook (Vrinnevi Hospital, Norrköping), Carl-Eric Nordgren County Hospital, Kalmar) and Göran Wallin and Anders Thorell (Karolinska Hospital, Stockholm).

We acknowledge the excellent help provided by the late secretary Gunnel Nordberg and statistician Lennart Gustafsson.

The study was supported by the Health Research Council of Southeast Sweden.

\section{References}

I. Charlson ME, Horwitz RI: Applying results of randomised trials to clinical practice: impact of losses before randomisation. BMJ 1984, 289: I28I-I289.

2. Moher D, Schulz KF, Altman DG: The CONSORT statement: revised recommendations for improving the quality of reports of parallell-group randomised trials. Lancet 200I, 357:1191-1194.

3. Livingston $\mathrm{EH}$, Rege RV: A nationwide study of conversion from laparoscopic to open cholecystectomy. Am J Surg 2004, I 88:205-2II.

4. McMahon AJ, Fischbacher CM, Frame SH, MacLeod CM: Impact of laparoscopic cholecystectomy: a population-based study. Lancet 2000, 356:1632-1637.

5. Nilsson E, Fored M, Granath F, Blomqvist P: Cholecystectomy in Sweden 1987-1999. A nationwide study of mortality and preoperative admissions. Scand J Gastroenterol 2005, 40: |478- | 485.

6. Majeed AW, Troy G, Nicholl JP, Smythe A, Reed MWR, Stoddard CJ, Peacock J, Johnson AG: Randomised, prospective, single-blind comparison of laparoscopic versus small-incision cholecystectomy. Lancet 1996, 347:989-994.

7. Ros A, Gustafsson L, Krook H, Nordgren C-E, Thorell A, Wallin G, Nilsson E: Laparoscopic cholecystectomy versus minilaparotomy cholecystectomy. A prospective, randomised, single-blind study. Ann Surg 200 I, 234:74I-749.

8. Nilsson E, Ros A, Rahmqvist M, Bäckman K, Carlsson P: Cholecystectomy: costs and health-related quality of life: a comparison of two techniques. Int J Qual Health Care 2004, 1 6:473-482.

9. Clavien P-A, Sanabria JR, Strasberg SM: Proposed classification of complications of surgery with examples of utility in cholecystectomy. Surgery 1992, I I I:5 18-526.

10. Jørgensen T: Treatment of gallstone patients. Copenhagen: National Institute of Public Health, Denmark, and Danish Institute for Health Technology Assessment; 2000. ISBN 87-9095I-44-I
II. Assalia A, Kopelman D, Hashmonai M: Emergency minilaparotomy cholecystectomy for acute cholecystitis: prospective randomized trial - implications for the laparoscopic era. World J Surg 1997, 2 I:534-539.

12. Assalia A, Schein M, Kopelman D, Hashmonai M: Minicholecystectomy vs conventional cholecystectomy: a prospective randomized trial - implications in the laparoscopic era. World J Surg 1993, 1 7:755-759.

13. O'Dwyer PJ, McGregor JR, McDermott EWM, Murphy JJ, O'Higgins $\mathrm{NJ}$ : Patient recovery following cholecystectomy through a 6 $\mathrm{cm}$ or $15 \mathrm{~cm}$ transverse subcostal incision: a prospective randomized clinical trial. Postgrad Med J 1992, 68:817-819.

14. Calvert NW, Troy GP, Johnson AG: Laparoscopic cholecystectomy: a good buy? A cost comparison with small-incision (mini) cholecystectomy. Eur J Surg 2000, I 66:782-786.

15. Leo J, Filipovic G, Krementsova J, Norblad R, Söderholm R, Nilsson $E$ : Open cholecystectomy for all patients in the era of laparoscopic cholecystectomy: a prospective cohort study. BMC Surgery 2006, 6:5.

16. Seale AK, Ledet WP: Minicholecystectomy: a safe, cost-effective day surgery procedure. Arch Surg 1999, I34:308-310.

17. Rozsos I, Ferenczy J, Schmitz R: Micro- and minicholecystectomy in the 2 I st century. Orv Hetil 2003, | 44:|29|-I297.

18. Oyogoa SO, Komenaka K, Ilkhani R, Wise L: Mini-laparotomy cholecystectomy in the era of laparoscopic cholecystectomy: a community-based hospital perspective. Am Surg 2003, 69(7):604-607.

19. Syrakos T, Antonitsis P, Zacharakis E, Takis A, Manousari A, Bakogiannis K, Efthimiopolous G, Achoulias I, Trikoupi A, Kiskinis D: Small incision (mini-laparotomy) versus laparoscopic cholecystectomy: a retrospective study in a university hospital. Langenbecks Arch Surg 2004, 389: I 72- I 77.

20. Devereaux PJ, Bhandari M, Clarke M, Montori VM, Cook DJ, Yusuf S, Sackett DL, Cinà CS, Walter SD, Haynes B, Schunemann HJ, Norman $\mathrm{GR}$, Guyatt GH: Need for expertice based randomised clinical trials. BMJ 2005, 330:88-93.

\section{Pre-publication history}

The pre-publication history for this paper can be accessed here:

http://www.biomedcentral.com/1471-2482/6/17/prepub

Publish with Biomed Central and every scientist can read your work free of charge

"BioMed Central will be the most significant development for disseminating the results of biomedical research in our lifetime. "

Sir Paul Nurse, Cancer Research UK

Your research papers will be:

- available free of charge to the entire biomedical community

- peer reviewed and published immediately upon acceptance

- cited in PubMed and archived on PubMed Central

- yours - you keep the copyright
BiolMedcentral 